\title{
Comparison between the 10- and the 30-s-long Wingate Anaerobic Test in summer Paralympic athletes with a lower limb impairment
}

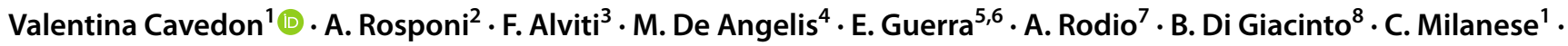 \\ M. Bernardi ${ }^{9,10}$
}

Received: 23 October 2019 / Accepted: 10 October 2020 / Published online: 3 December 2020

(c) The Author(s) 2020

\begin{abstract}
Background The 30-s-long Wingate Anaerobic Test (WAnT_30s) has some limitations in high-level athletes. A shorter version might be helpful for both clinical applications and performance assessment. The comparison between the traditional WAnT_30s and a shorter version has never been carried out yet in Paralympic athletes.

Aim To assess if a 10-s-long Wingate Anaerobic Test (WAnT_10s) could be used to accurately assess and predict the anaerobic components of physical fitness as an alternative to the traditional WAnT_30s in male Paralympic athletes.

Methods Forty-four trained male Paralympic Athletes grouped by severity of locomotor impairment completed the WAnT_30s and the WAnT_10s with an arm cranking ergometer. Differences between mean and peak power achieved throughout both WAnTs were analysed using a mixed-design analysis of variance and predictivity was assessed by stepwise linear regression analysis.

Results In the whole sample, peak power values were similar $(P>0.005)$ in the two tests and the WAnT_10s mean power was significantly higher than that in the WAnT_30s $(P<0.005)$. Finally, the mean power measured during WAnT_30s showed high level of predictability from mean power measured during WAnT_10s and the Functional class (adjusted $R^{2}=0.906$; $P<0.001$ ).

Conclusion The WAnT_10s is accurate to assess peak power, is definitively appropriate to evaluate the alactic anaerobic metabolism and seems able to predict the mean power as traditionally evaluated through a WAnT_30s in male Paralympic Athletes. Thus, it can be used to assess the anaerobic components of physical fitness in this athletic population.
\end{abstract}

Keywords Adapted sports · Anaerobic capacity $\cdot$ Anaerobic power $\cdot$ Anaerobic physical fitness evaluation $\cdot$ Physical disability

\section{Abbreviations}

WAnT Wingate Anaerobic Test

WAnT_30s 30-s-long Wingate Anaerobic Test
WAnT_10s 10-s-long Wingate Anaerobic Test ISMS Institute of Sports Medicine and Science CONI Italian National Olympic Committee
Valentina Cavedon

valentina.cavedon@univr.it

1 Department of Neurosciences, Biomedicine and Movement Sciences, University of Verona, Piazzale L.A. Scuro, 10, 37134 Verona, Italy

2 Sports Medicine Service, Provincial Agency for Health of the Autonomous Province of Trento, Trento, Italy

3 Department of Anatomy, Histology, Forensic Medicine and Orthopedics, Board of Physical Medicine and Rehabilitation "Sapienza", University of Rome, Rome, Italy

4 Department of Applied Clinic Sciences and Biotechnology, University of L'Aquila, L'Aquila, Italy
5 Sports Medicine Service, Local Health Unit of Modena, Modena, Italy

6 Local Health Unit of Roma, ASL Roma 1, Rome, Italy

7 Department of Human, Social and Health Sciences, University of Cassino and Southern Lazio, Rome, Italy

8 Institute of Sport Medicine and Science, Sport and Health, Rome, Italy

9 Department of Physiology and Pharmacology "V. Erspamer", School of Specialty in Sports Medicine and Physical Exercise, "Sapienza" University of Rome, Rome, Italy

10 Italian Paralympic Committee, Rome, Italy 


\begin{tabular}{|c|c|}
\hline NOVA & Analysis of variance \\
\hline$\eta_{p}^{2}$ & Partial eta squared \\
\hline$r$ & $\begin{array}{l}\text { Pearson's product-moment correlation } \\
\text { coefficient }\end{array}$ \\
\hline$R^{2}$ & Adjusted coefficient of determination \\
\hline SEE & Standard error of the estimate \\
\hline$f^{2}$ & Cohen's $f$ squared \\
\hline RMSPE & Root mean squared prediction error \\
\hline ATP & Adenosine triphosphate \\
\hline SCI & Spinal cord injury \\
\hline AMP & Amputation \\
\hline ILG & Groups of level of impairment \\
\hline $\mathrm{VO}_{2 \text { peak }}$ & Oxygen uptake peak \\
\hline
\end{tabular}

\section{Introduction}

The Paralympic Games represent one of the major international mega-sport events in the early 21 st century [1]. Following to the advancement of the Paralympic movement over the last two decades, the physiological monitoring of Paralympic athletes is now common practice [2] and today the intensity and the energy expenditure of the practiced sport and the physical fitness evaluations are considered fundamental in the assessment of athletes with physical impairments [3, 4].

Upper limb anaerobic power and capacity are fundamental components of physical fitness in both able-bodied [5] and Paralympic athletes [4, 6]. In fact, performance in many individual and team Paralympic sport carried out by individuals with a locomotor impairment is considerably dependent on maximal anaerobic power and capacity $[4,6$, 7]. In particular, anaerobic power is a performance-related physical fitness component among many individual sports, such as for example, track events (short distance) and field events (throws and both high and long jumps) in athletics, para alpine skiing (for athletes with either physical or visual or intellectual impairment) and, among the team sports, wheelchair basketball, sitting volleyball and Para ice hockey (formerly called ice sledge hockey). Anaerobic capacity is a physical fitness prerequisite of wheelchair track event sprints (up to $400 \mathrm{~m}$ ), track cycling, swimming (short distances), alpine skiing, and is a limiting factor in all above quoted team sports. Furthermore, it has been shown that the assessment of these components of physical fitness is of great importance not only for competitive success in Paralympic sports [4] but also for health-related and quality-oflife-related reasons [6, 8, 9]. Indeed, many motor activities of daily living conducted by individuals with a locomotor impairment are performed in a short duration and utilize anaerobic metabolism [8, 9]. For these reasons, monitoring the anaerobic components of physical fitness in Paralympic athletes is useful to evaluate training status, to prevent overtraining, to plan appropriate training protocols maximizing their benefits $[4,6,10]$ and to guarantee an appropriate health status and quality of life [8, 9].

Classical interpretation of the anaerobic components of physical fitness is based on the rationale that the fuel for an anaerobic performance comes from two primary energy sources: an "alactic" anaerobic metabolism with high power and low capacity (largely phosphagen based and typically depleted within 6-9 s) and an anaerobic glycolytic metabolism with lower power and larger capacity (mainly limited by hydrogen ion accumulation and the consequent acidosis, which is exhausted within about 45 s) [10-13]. Power and capacity of each single energy metabolism system in providing adenosine triphosphate (ATP) are strictly interrelated [14, 16, 17], indeed maximal turnover rate (mmol/ $\mathrm{kg}$ of wet muscle per second) and total human body stores ( $\mathrm{mmol} / \mathrm{kg}$ of wet skeletal muscle) are equal to 3-6 and 20-25 for ATP-phosphocreatine (a-lactic anaerobic metabolism) and 1.5-3 and 50 for anaerobic glycolysis (lactic anaerobic metabolism), respectively [14, 16]. Furthermore, in a supramaximal (intensity over the maximal aerobic power) all-out exercise lasting $30 \mathrm{~s}$, the mean ATP turnover rate of the aerobic metabolism, contributing for about $9 \%$ of the total energy supplied to complete the effort, reaches in $6 \mathrm{~s}$ values of about $1.32 \mathrm{mmol} / \mathrm{kg}$ of dry muscle per second [16], corresponding to about 0.4 per $\mathrm{kg}$ of wet muscle per second.

There are many laboratory procedures for evaluating directly [5, 17-21] and indirectly [4, 6, 8, 22-24] the anaerobic components of physical fitness. The indirect procedures, in which the anaerobic evaluation is based on the mechanical performance of the athlete on different ergometers, are definitively more popular [4, 6, 22-24]. Among the latter, the Wingate Anaerobic Test (WAnT), an all-out test in which the ergometer allows that the prefixed torque is maintained constant and the individual pedals at her/his maximal speed, has been adapted since the 1980s for individuals with locomotor impairments using arm cranking ergometers [24, 25]. The WAnT has many versions, of which the 30-s-long test (WAnT_30s) has been applied most often in athletes and non-athletes with different physical impairments [6, 22, 23, 26-37]. On the other hand, shorter WAnT protocols have also been employed in studies dealing with high-level athletes with physical impairments [4, 38].

Scientific data [20, 39, 40] documented some limitations in the use of the WAnT_30s in able-bodied individuals suggesting abbreviated versions of the WAnT_30s, e.g. 20-, 10or 6-s-long protocols. These limitations of the WAnT_30s were reported in high-level athletes [20, 40] and included detrimental physical side effects of the test, such as headache, vomiting, dizziness, and nausea [39, 41], leading to less than maximal efforts during repeated testing. Furthermore, shorter versions of the WAnT would be useful to those athletes requiring for their performance short bursts 
of explosive power because during a WAnT_30s there is a predominance of phosphagen breakdown (a-lactic anaerobic metabolism) in the first 7-9 s and a predominance of anaerobic glycolysis (lactic anaerobic metabolism) from the 10th second to the 30th second of this maximal effort [16, 42, 43]. In analogy with the able-bodied literature, it is, therefore, reasonable to assume that shortening the traditional WAnT_30s might be helpful in Paralympic athletes from both performance-related and clinical applications. To the best of our knowledge, the results obtained in WAnT_30s and in a shorter version of the WAnT (e.g., WAnT_10s) have never been compared yet in Paralympic athletes. Accordingly, this research was conducted on a sample of 44 male Paralympic athletes with a twofold aim. First, to assess possible differences among "anaerobic" mechanical power and capacity variables assessed with the traditional WAnT_30s and an abbreviated (10-s long) WAnT (WAnT_10s). Due to the heterogeneity of the considered population, the extent of functional limitation of Paralympic athletes has been also considered. Second, to explore whether the mean power assessed in the WAnT_10s could be used in Paralympic athletes to accurately predict the mean power of the traditional WAntT_30s.

\section{Methods}

\section{Participants}

Forty-four trained male Paralympic Athletes with a locomotor impairment were tested before their participation in the Summer Paralympic Games. Paralympic athletes were engaged in wheelchair basketball $(n=12$, including 7 players with a thoracic spinal cord injury (SCI), 1 with lower limb poliomyelitis, 2 with trans-tibial amputation (AMP), 1 with trans-femoral AMP, 1 with a health condition determining a reduced knee range of movement), wheelchair tennis $(n=5$, including 3 tennis players with an incomplete cervical SCI,
1 with a thoracic SCI and 1 with a lumbar SCI), para tabletennis $(n=3$, including 1 with a thoracic SCI, 1 with a hemiparesis from cerebral palsy and 1 with a health condition determining reduced knee and ankle ranges of movement), hand-bike ( $n=1$, with an incomplete lumbar SCI), paraswimming $(\mathrm{n}=4$, including 1 with a complete cervical SCI, 1 with an incomplete cervical SCI, 1 with trans-tibial amputation, 1 with a hemiparesis from cerebral palsy), shooting ( $n=4$, including 3 with a thoracic SCI, 1 with lower limb poliomyelitis), archery ( $n=3$, including 1 with spina bifida, 1 with tetra-paresis from cerebral palsy, 1 with lower limb poliomyelitis), sailing ( $n=2$, including 1 with an incomplete cervical SCI and 1 with complete thoracic SCI), Para athletics $(n=6,4$ competing in field events, including 2 with a complete thoracic SCI, 1 with trans-tibial amputation, 1 with lower limb poliomyelitis and 2 competing in field events, both with complete cervical SCI) and wheelchair fencing ( $n=4$, including 3 with complete thoracic SCI and 1 with incomplete lumbar SCI). Athletes were pooled, in accordance with the classification system adopted in Para swimming [44], into three groups of level of impairment (ILG). In this Paralympic classification, the functional activity is assessed through an ordinal scale ranging from 1 to 10 (the lower the number, the more severe the activity limitation). The three groups were formed as follows: ILG-A $(n=9)$, which included classes from 2 to 4; ILG-B $(n=22)$ which included classes from 5 to 7 ; ILG-C $(n=13)$, which included classes from 8 to 10 . Table 1 shows, for the whole sample of Paralympic Athletes divided in accordance with the ILG (A-B-C), age, main anthropometric characteristics (body mass and stature), health condition, para-swimming class, sport disciplines and oxygen uptake peak (aerobic fitness). All data used to characterize the participants of the present study were obtained through a retrospective chart review of Paralympic athletes selected to compete in Athens 2004 [45]. $V \mathrm{O}_{2 \text { peak }}$ had been assessed through standard measurements using a breath-by-breath metabolimeter (Quark $b^{2}$,

$V \mathrm{O}_{2 \text { peak }}$ ) of Paralympic athletes divided by group of level of impairment (ILG-A, ILG-B and ILG-C)
Table 1 Age, main anthropometric characteristics (body mass and stature), para-swimming functional classes (FC), health condition (HC), sport disciplines and aerobic fitness (oxygen uptake peak-

\begin{tabular}{lllllll}
\hline & Age (years) & Body mass $(\mathrm{kg})$ & Stature $(\mathrm{m})$ & $\mathrm{FC}($ from 2 to 10) & $V_{2 \text { peak }}\left(\mathrm{ml} \mathrm{kg}^{-1} \mathrm{~min}^{-1}\right)$ & $\mathrm{HC}$ \\
\hline $\begin{array}{l}\text { ILG-A (classes 2-3-4) } \\
(n=9)\end{array}$ & $37.00(10.64)$ & $66.67(10.00)$ & $1.78(0.05)$ & $3.44(0.73)$ & $20.69(7.42)$ & $8 \mathrm{SCI}, 1 \mathrm{CP}$ \\
$\begin{array}{l}\text { ILG-B (classes 5-6-7) } \\
(n=22)\end{array}$ & $35.41(5.20)$ & $72.50(11.43)$ & $1.78(0.11)$ & $5.82(0.66)$ & $27.16(6.25)$ & $19 \mathrm{SCI}, 1 \mathrm{PL}, 1 \mathrm{SB}, 1 \mathrm{CP}$ \\
$\begin{array}{l}\text { ILG-C (classes 8-9-10) } \\
(n=13)\end{array}$ & $36.38(9.70)$ & $76.85(12.21)$ & $1.80(0.09)$ & $8.85(0.90)$ & $35.78(7.79)$ & $2 \mathrm{SCI}, 3 \mathrm{PL}, 5 \mathrm{AMP}, 2$ \\
\hline
\end{tabular}

Data are mean (standard deviation)

$S C I$ spinal cord injury, $P L$ poliomyelitis, $S B$ spina bifida, $A M P$ amputation, $C P$ Cerebral palsy, LA Les Autres, meaning "other" health conditions different from SCI, PL, SB, AMP, CP 
Cosmed, Italy) during an arm cranking incremental maximal exercise test carried out up to exhaustion [46].

All testing procedures were approved by the Review Board of the Institute of Sports Medicine and Science (ISMS) of the Italian National Olympic Committee (CONI) (Rome, Italy) for athlete testing and by "Santa Lucia Foundation" Ethical Committee. All participants signed informed consent form after they had been fully informed about the purposes and the testing procedures of the study.

\section{Procedures}

Functional data related to the assessment of anaerobic fitness, collected during the institutional health and fitness screenings [47] funded by the Italian Paralympic Committee, carried out, as primary clinical care, in the ISMS of CONI to assess a risk-free sport eligibility [45] before the Summer Paralympic Games, were analysed and presented in the current study.

All Paralympic Athletes completed the WAnT_30s and the WAnT_10s with an electrically braked ergometer (excalibur lode, Groningen, Holland) positioned on a specific table fixed to the ground to prevent any movement of the ergometer during the test.

This study adopted a randomized 2-sequence (AB | BA), 2-tests (WAnT_30s vs. WAnT_10s) crossover design. At the beginning of the study, the 44 study participants were randomly assigned to one of the two sequences using the Wei's urn design, a randomization technique for balancing treatment assignments [48]. Participants randomized to the AB sequence performed a WAnT_30s and after a washout period of $1.5 \mathrm{~h}$, a WAnT_10s. Participants randomized to the BA sequence performed a WAnT_10s and after $1 \mathrm{~h}$ a WAnT_30s.

Considering the severity of the impairment (class) and the aerobic fitness status (see Table 1), the best performing torque factor was established in the day preceding the data collection [37]. Athletes performed three to five WAnT_10s at different torque factors (ranging from 0.1 to 0.9 ) to identify the torque factor that allowed them to achieve the highest mean power values [49]. For each athlete, the torque value $(\mathrm{N} \mathrm{m})$ was then set up by multiplying the body mass $(\mathrm{kg})$ by the torque factor. In this study, the torque factors ranged from 0.3 to 0.7 (Table 2). After a 15 -min standardized submaximal warm-up at a power ranging between 10 and 50 watts depending on the level of the lesion and the physical fitness status, athletes were submitted to tests. They were requested to crank at a very low power (range between 5 and 20 watts) and at less than 30 revolutions per minute and then, after a countdown of $10 \mathrm{~s}$, the constant torque was applied and they were requested to start cranking at their maximum speed trying to maintain the crank rate as high as possible until the end of the test. The mean and the peak power (both expressed in $\mathrm{W} \mathrm{kg}^{-1}$ ) achieved throughout the WAnT_30s and the WAnT_10s were considered for analysis.

\section{Statistical analysis}

In this work, an ABIBA crossover design was adopted. Numerical variables were summarized using mean \pm standard deviation and normal distribution of the data was tested using the Shapiro-Wilk test. All variables were normally distributed. Accordingly, parametric statistics were adopted. Descriptive statistics (mean and standard deviation) were computed for all variables using standard procedures. Differences between mean and peak power registered in the WAnT_30s and WAnT_10s tests and possible interaction between athletes' functional limitation were analysed using mixed-design analysis of variance (ANOVA) with the three ILGs (A, B and C) and the two tests (WAnT_30s and WAnT_10s) with repeated measures on the second factor. When the repeated measures factor violated the assumption of sphericity $(P<0.05)$, the Greenhouse-Geisser correction, which refers to degrees of freedom of F statistics, was used. For each ANOVA model, if significant effects were detected, post hoc analysis with Bonferroni's correction was carried out. Cohen's partial eta squared $\left(\eta_{\mathrm{p}}^{2}\right)$ was used to calculate the effect size in the ANOVA with repeated measures. According to Cohen's guidelines [50], effect size values

Table 2 Wingate Anaerobic Test (WAnT): mean power and peak power assessed in two protocols of different length for the whole-sample as well as for the three subgroups of different classes based on the level of impairment (ILG)

\begin{tabular}{llllll}
\hline & Torque factor & $\begin{array}{l}\text { WAnT_30s } \\
\text { Mean power }\left(\mathrm{W} \mathrm{kg}^{-1}\right)\end{array}$ & $\begin{array}{l}\text { WAnT_10s } \\
\text { Mean power }\left(\mathrm{W} \mathrm{kg}^{-1}\right)\end{array}$ & $\begin{array}{l}\text { WAnT_30s } \\
\text { Peak power }\left(\mathrm{W} \mathrm{kg}^{-1}\right)\end{array}$ & $\begin{array}{l}\text { WAnT_10s } \\
\text { Peak power }(\mathrm{W} \mathrm{kg}\end{array}$ \\
\hline Whole-sample $(n=44)$ & $0.50(0.16)$ & $4.00(1.36)$ & $4.51(1.70)$ & $5.87(1.98)$ & $5.79(2.08)$ \\
ILG-A $(n=9)$ & $0.32(0.14)$ & $2.18(0.78)$ & $2.26(1.04)$ & $3.29(1.15)$ & $3.01(1.33)$ \\
ILG-B $(n=22)$ & $0.49(0.11)$ & $3.92(0.82)$ & $4.43(0.95)$ & $5.69(1.14)$ & $5.68(1.15)$ \\
ILG-C $(n=13)$ & $0.64(0.09)$ & $5.39(0.67)$ & $6.22(1.05)$ & $7.97(1.06)$ & $7.90(1.19)$ \\
\hline
\end{tabular}

Data are mean (standard deviation)

WAnT_30s 30-s-long Wingate Anaerobic Test, WAnT_10s 10-s-long Wingate Anaerobic Test 
were interpreted as small $\left(\eta_{p}^{2}=0.01\right)$, medium $\left(\eta_{p}^{2}=0.06\right)$, and large $\left(\eta_{\mathrm{p}}^{2}=0.14\right)$.

A stepwise linear regression was run to determine the accuracy with which the mean power assessed during the WAnT_30s could be predicted from the values of the mean power assessed during the WAnT_10s and the Functional class. Adjusted coefficients of determination $\left(R^{2}\right)$, standard error of the estimate (SEE) and residual diagnostics were used to represent the goodness of the predictor model. The Durbin-Watson test, the variance inflation factor, the tolerance value, and the condition index were calculated to test collinearity. For the regression model, Cohen's $f$ squared $\left(f^{2}\right)$ was used to calculate the effect size of the regression model and interpreted according to Cohen's guidelines [50]. The developed regression model was then validated using a repeated tenfold cross-validation (with 1000 replications), estimating for each cross-validation sample the root mean squared prediction error (RMSPE) and the $R^{2}$.

Statistical power of the sample was evaluated using G*Power Software 3.1 [51] on the basis of the observed effect sizes. All analyses were performed with SPSS v. 16.0 (IBM Corp., Armonk, NY, USA) and R-3.6.2 (Foundation for Statistical Computing, Vienna). The alpha value was set at 0.05 .

\section{Results}

Descriptive statistics (mean and standard deviation) of the mean and peak power in the WAnT_30s and in the WAnT_10s for the three ILGs (A, B and C) and the aggregate sample are summarized in Table 2. For the same samples, Table 2 also shows the multiplying factor (torque factor) of the body mass used to obtain the torque use to carry out the test.

\section{Mixed-design analysis of variance (ANOVA)}

The results of the mixed-design ANOVA with the three ILGs (A, B and C) and the two tests (WAnT_30s and WAnT_10s) with repeated measures on the second factor showed a significant main effect of ILG on both peak power $(F=47.374$, $\left.P<0.001, \eta_{\mathrm{p}}{ }^{2}=0.698\right)$ and mean power $(F=47.547$, $P<0.001, \eta_{\mathrm{p}}{ }^{2}=0.956$ ). Post hoc analysis with Bonferroni's correction revealed that ILG-A had statistically significant lower values of both peak and mean power than ILG-B $(P<0.001$ for both $)$ and ILG-C $(P<0.001$ for both). The mean values of peak and mean power were also significantly lower in ILG-B than in ILG-C $(P<0.001$ for both).

The results of mixed-design ANOVA showed a statistically significant main effect of Wingate test condition for mean power $\left(F=28.318, P<0.001 ; \eta_{\mathrm{p}}{ }^{2}=0.408\right)$, while no significant main effect of test condition was found for peak power $\left(F=2.828, P=0.100, \eta_{\mathrm{p}}{ }^{2}=0.065\right)$. In the whole sample, the mean power assessed in the WAnT_30s was lower $(-12.8 \%)$ in comparison with the mean power assessed in the WAnT_10s.

A statistically significant main effect for ILG by Wingate test condition interaction was found for mean power $\left(F=4.976, P=0.012, \eta_{\mathrm{p}}{ }^{2}=0.195\right)$, while no significant main effect for ILG by test condition interaction was found for peak power $\left(F=1.155, P=0.325, \eta_{\mathrm{p}}{ }^{2}=0.053\right)$. Post hoc analysis with Bonferroni's correction revealed that the values of mean power assessed in the WAnT_30s were significantly lower than those assessed in the WAnT_10s in both ILG-B and ILG-C ( $P<0.001$ for both). In ILG-A (athletes with either tetraplegia or tetraparesis), the values of mean power were similar in the two Wingate tests $(P=0.673)$. For each ANOVA model, post hoc power analysis exceeded 0.99 .

Table 3 shows the same variables dividing the whole sample by the practiced sport. Athletes with a locomotor impairment, without impaired upper limb, competing in intermittent (circumstance) sports (those with alternate anaerobic/aerobic metabolisms), including wheelchair basketball, wheelchair fencing and wheelchair tennis, showed relative $\left(\mathrm{W} \mathrm{kg}^{-1}\right)$ peak and mean power values in the WAnT_10s and WAnT_30s equal to $6.34 \pm 1.47$ and $4.98 \pm 1.25$, and $6.31 \pm 1.33$ and $4.43 \pm 0.91$, respectively. Athletes with a locomotor impairment competing in endurance sports (excluding those with tetraplegia), including hand-bike and swimming, showed relative $\left(\mathrm{W} \mathrm{kg}^{-1}\right)$ peak and mean power values in the WAnT_10s and WAnT_30s equal to $7.29 \pm 1.36$ and $5.46 \pm 0.64$, and $8.07 \pm 1.17$ and $5.61 \pm 0.56$, respectively. Athletes with a locomotor impairment (excluding those with either tetraplegia or tetraparesis) competing in skill sports, including archery, table tennis, sailing and shooting, showed relative $\left(\mathrm{W} \mathrm{kg}^{-1}\right)$ peak and mean power values in the WAnT_10s and WAnT_30s equal to $6.13 \pm 1.27$ and $4.79 \pm 1.12$, and $6.00 \pm 1.16$ and $3.94 \pm 0.87$, respectively.

\section{Regression analysis}

In the whole sample of Paralympic athletes, the mean power assessed in the WAnT_30s showed high level of predictability from mean power assessed in the WAnT_10s and the functional class (adjusted $R^{2}=0.906, \mathrm{SEE}=0.416$, $\left.f^{2}=9.638 ; P<0.001\right)$. A scatter plot and the corresponding regression line showing the relationship between mean power in WAnT_30s ( $y$ axis) and the mean power in WAnT_10s ( $x$ axis) is reported in Fig. 1a. The model for the prediction of the mean power in the WAnT_30s was: mean power (WAnT_30s) $=0.611$ (mean power assessed in the WAnT_10s) + 0.137 (functional class) +0.393 . 
Table 3 Wingate anaerobic test (WanT). Mean power and peak power assessed in two protocols of different length dividing the group by the practiced sport

\begin{tabular}{|c|c|c|c|c|c|}
\hline $\begin{array}{l}\text { Sport (number of Paralym- } \\
\text { pic athletes) }\end{array}$ & $\mathrm{FC}$ (from 2 to 10 ) & $\begin{array}{l}\text { WAnT_30s (mean } \\
\text { power } \mathrm{W} \mathrm{kg}^{-1} \text { ) }\end{array}$ & $\begin{array}{l}\text { WAnT_10s (mean } \\
\text { power } \mathrm{W} \mathrm{kg}^{-1} \text { ) }\end{array}$ & $\begin{array}{l}\text { WAnT_30s (peak } \\
\text { power } \mathrm{W} \mathrm{kg}^{-1} \text { ) }\end{array}$ & $\begin{array}{l}\text { WAnT_10s } \\
\text { (peak power } \\
\mathrm{W} \mathrm{kg}^{-1} \text { ) }\end{array}$ \\
\hline Archery (2) & $7.5(0.71)$ & $5.10(0.82)$ & $6.34(1.38)$ & $7.36(0.84)$ & $7.83(1.38)$ \\
\hline Archery tetra $(1)^{\mathrm{a}}$ & 3.0 & 1.44 & 1.98 & 3.75 & 3.04 \\
\hline Field events (4) & $7.25(2.22)$ & $5.13(1.53)$ & $6.09(2.18)$ & $7.76(2.85)$ & $7.59(2.67)$ \\
\hline Track events tetra $(2)^{\mathrm{b}}$ & $2.5(0.71)$ & $1.41(0.45)$ & $1.03(0.2)$ & $1.97(0.87)$ & $1.28(0.06)$ \\
\hline Hand bike (1) & 8 & 5.00 & 4.88 & 6.80 & 6.22 \\
\hline Sailing (1) & 5 & 3.60 & 4.33 & 5.53 & 5.67 \\
\hline Sailing tetra $(1)^{\mathrm{b}}$ & 4 & 2.47 & 2.83 & 3.45 & 3.33 \\
\hline Shooting (4) & $6.25(1.26)$ & $3.68(0.80)$ & $4.59(0.91)$ & $5.83(1.33)$ & $5.83(1.15)$ \\
\hline Swimming (2) & $8.5(2.12)$ & $5.91(0.28)$ & $5.75(0.55)$ & $8.71(0.52)$ & $7.83(1.41)$ \\
\hline Swimming tetra $(2)^{\mathrm{b}}$ & $3.5(0.71)$ & $2.87(1.21)$ & $2.98(1.47)$ & $3.72(1.76)$ & $3.65(1.99)$ \\
\hline Para table tennis (3) & $7.3(1.53)$ & $3.61(0.61)$ & $4.19(0.56)$ & $5.47(0.76)$ & $5.54(0.78)$ \\
\hline WC basketball (12) & $7.0(1.91)$ & $4.49(1.05)$ & $4.93(1.53)$ & $6.28(1.60)$ & $6.28(1.79)$ \\
\hline WC fencing (4) & $5.75(0.96)$ & $4.01(0.50)$ & $4.92(0.39)$ & $6.05(0.43)$ & $6.27(0.45)$ \\
\hline WC tennis (2) & $7.0(1.41)$ & $4.88(0.32)$ & $5.36(0.60)$ & $7.00(0.43)$ & $6.84(0.68)$ \\
\hline WC tennis tetra $(3)^{\mathrm{b}}$ & $4(0)$ & $2.40(0.39)$ & $2.51(0.93)$ & $3.68(1.06)$ & $3.61(1.07)$ \\
\hline
\end{tabular}

Para-swimming functional Classes (FC) are also included. Data are mean (standard deviation)

WAnT_30s 30-s-long Wingate Anaerobic Test, WAnT_10s 10-s-long Wingate Anaerobic Test, WC Wheelchair

${ }^{\mathrm{a}}$ Tetraparesis from cerebral palsy

${ }^{\mathrm{b}}$ Tetraplegia from spinal cord injury

Residual diagnostics suggested that residuals are distributed around 0 and no unexplained (linear/nonlinear) structure can be detected in the residuals (Fig. 1b). The distribution of residuals is also pretty close to a Gaussian distribution (Fig. 1c and Fig. 1d).

The Durbin-Watson test, the variance inflation factor, the condition index, and tolerance value were equal to 2.2 , $1.0,5.5$ and 1.0, respectively, showing that they were robust to collinearity. Post hoc power analysis in the regression analysis exceeded 0.99 , suggesting a good model sensitivity to Type II error as well as that the model was adequately powered to detect the true effect of the predictor variable. The validation of our model using a repeated tenfold crossvalidation, showed that the model has good predictive performance $(\mathrm{RMSPE}=$ mean 0.43 and standard error $=0.005$; $R^{2}=$ mean 0.89 and standard error $=0.002$ ).

\section{Discussion}

The assessment of anaerobic components of physical fitness is of great importance in Paralympic sports for both performance and health-related reasons $[4,6,14,16,16,17,17$, 21, 27, 28, 35]. The traditional 30-s-long Wingate Anaerobic Test (Want_30s) has been widely used to evaluate the anaerobic performance in individuals with locomotor impairment $[6,23,27-37]$. In the present research, we studied 44 elite athletes with a locomotor impairment deriving from the main typical health conditions common among Paralympic participants and competing in sports with a wide range of energy expenditure and intensities $[49,52]$ and, therefore, with a wide range of aerobic fitness [4, 53].

We evaluated the hypothesis, to our best knowledge not yet tested, that a short version of the WAnT, i.e. a 10-s-long Wingate Anaerobic Test (WAnT_10s), could be used to accurately assess and predict the anaerobic components of physical fitness as an alternative to the traditional WAnT_30s in Paralympic athletes. The results of the present study demonstrated that the WAnT_10s is both a valid tool for the evaluation of the upper-body anaerobic fitness and, through its mean value, accurately predicts the mean power assessed in the WAnT_30s.

The traditional WAnT_30s is routinely used to monitor training status in athletes with a locomotor impairment, such as wheelchair basketball players [37]. The assessment of the anaerobic components of physical fitness through an abbreviated version of the traditional WAnT_30s might be helpful in Paralympic athletes from both clinical (rehabilitation) and performance applications [4, 49]. Indeed, the WAnT_10s has been used to assess "mean explosive power" in Winter Paralympic Athletes [4, 49]. In the present study, we took into consideration that 1 ) the peak power (which should be the same in both tests because it should occur in the first ten seconds of a maximal effort exercise) is expression of the 

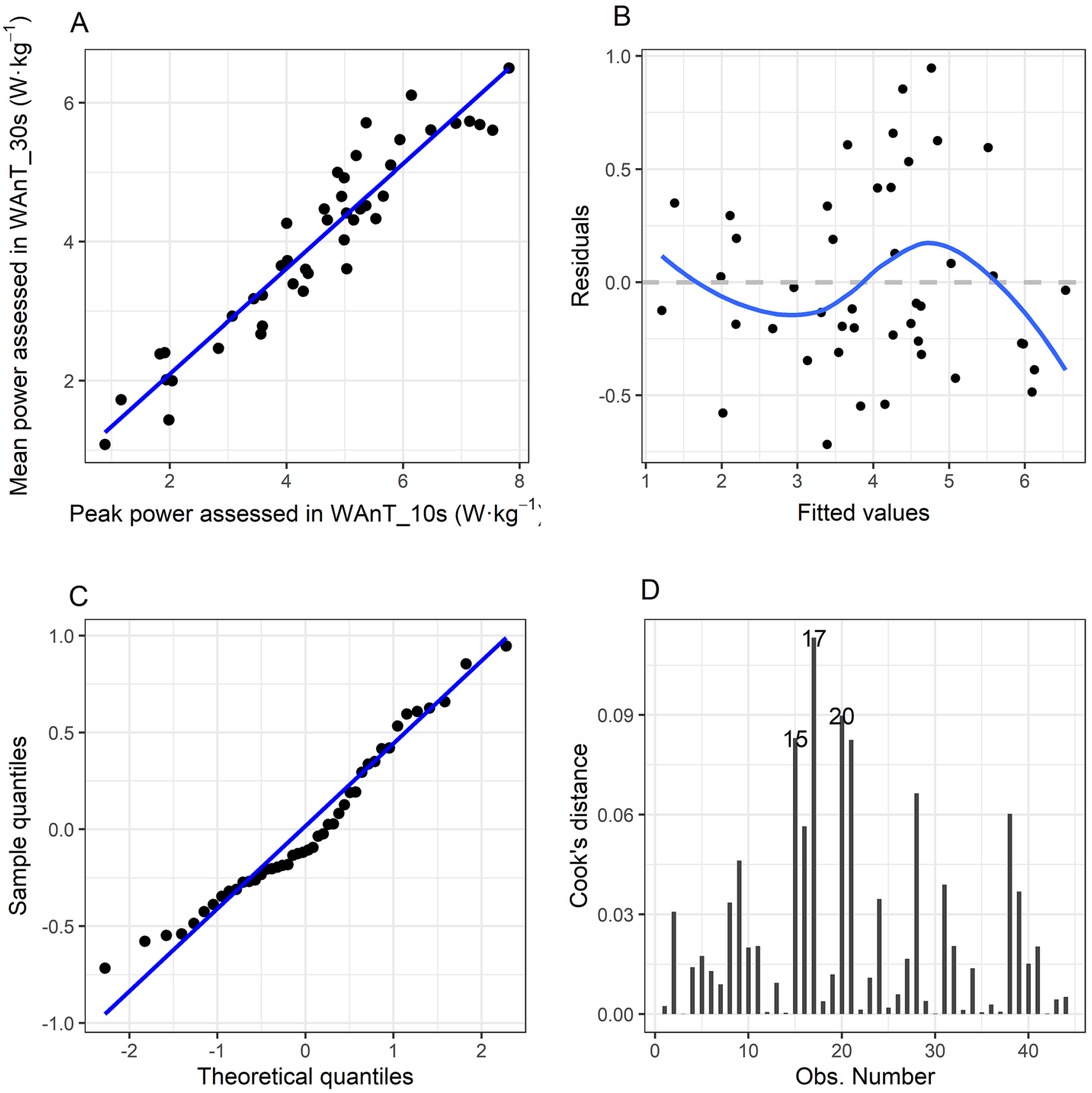

Fig. 1 Regression analysis. a Scatter plot and the corresponding regression line for the relationship between mean power in the WAnT_30s and mean power in the WAnT_10s. b Scatter plot of model residuals versus predicted values of WAnT_30s. c Scatter plot

ATP maximal turnover rate of (i.e., energy coming from) the phosphocreatine $[2,16,21]$, the lactic anaerobic metabolism contributes for about $45 \%$ in the ATP turnover in a 10-s-long all-out exercise test [17]. Therefore, in the anaerobic fitness functional assessment context, the WAnT_10s should be an appropriate test to evaluate not only the power, but also the capacity of the phosphagens, being the energy contribution from phosphocreatine null after $10 \mathrm{~s}$ of all-out exercise [54].

of the empirical quantiles of WAnT_30s versus the theoretical quantile of a Gaussian. d Scatter plot of the estimated Cook's distance for each observation. MP: mean power, WAnT_30s: 30-s-long Wingate Anaerobic Test, WAnT_10s: 10-s long Wingate Anaerobic Test

In the present study, the IPC classification system used for para-swimming [44] has been used to divide the athletes in three groups with different levels of impairment. The impact of this subdivision in groups (ILGs: A, B and C) in the peak and mean values of both WAnTs was statistically significant. Athletes pooled in the ILG-A, which included only those with tetraplegia or tetra-paresis, showed the lowest values, while the athletes pooled in the ILG-C, 
which included athletes with the lowest levels of impairment (deriving from either lumbar paraplegia, or lower limb poliomyelitis or trans-femoral and trans-tibial amputations or other less severe health conditions), showed the highest values. These results in both peak and mean power of both WAnTs are consistent with the smallest amount of muscle available in the athletes of the ILG-A and the lower amount of muscle available in the athletes of the ILG-B than in those of the ILG-C.

The results of the present study indicated that in the whole sample, the peak power output was similar in the two tests (i.e., a non-significant $0.08 \mathrm{~W} \mathrm{~kg}^{-1}$ difference between the WAnT_30s and the WAnT_10s was found). This result shows, as pointed out in the Literature and hypothesized in the present study, that Paralympic athletes achieved their peak power within the initial $10 \mathrm{~s}$ of the anaerobic exercise and that similar maximal effort was demonstrated over both tests, showing the good quality of the assessment in both WAnTs. This result was in line with a previous investigation [55] conducted on able-bodied participants and carried out on a Monark cycle ergometer reporting that the peak power assessed during the traditional WAnT_30s was similar to that assessed in a 20-s-long WAnT. Because peak power measured during a 10-s all-out test depends on the power (ATP turnover) of the alactic anaerobic metabolism [16, 21] and this energy metabolism is completely exhausted after $10 \mathrm{~s}$ [54], it is definitively reasonable to conclude that peak power values in both tests must be the same.

As expected, as a consequence of both fatigue and different power of alactic and lactic anaerobic metabolisms $[16,17,42,54]$, the results from this study indicated that an abbreviated version of the traditional WAnT_30 produced significantly higher relative mean power outputs. In fact, when considering the whole sample of Paralympic athletes $(n=44)$, the mean power assessed in the WAnT_10s was significantly higher (11.3\%) than the mean power assessed in the WAnT_30s. These results were in line with the previously quoted investigation [55] conducted on able-bodied participants reporting that the mean power assessed during a shorter version of the WAnT (i.e., a 20-s-long WAnT) was significantly higher $(+11.1 \%)$ than that measured in the traditional 30-s-long WAnT.

When assessing the comparisons in the anaerobic outputs between WAnT_30s and WAnT_10s according to the extent of functional limitation, the results of the present study highlighted no significant ILG by test interaction for peak power but a significant ILG by test interaction for mean power. In details, only group $\mathrm{B}$ and $\mathrm{C}$ produced greater mean power by, respectively, $+11.5 \%$ and $+13.3 \%$ in the WAnT_10s versus the WAnT_30s, likewise the trend reported by Laurent and colleagues [55] as assessed in the able-bodied on a Monark cycle ergometer. The fact that no difference in the mean power of the WAnTs of two different lengths was found in ILG-A (group which included athletes with either tetraplegia or tetraparesis) could be due to the reduced amount of muscles available for contraction and the consequent reduced contribution of the alactic anaerobic metabolism in both tests and in particular in the WAnT_10s.

In the present study, the athletes were divided by the practiced sports, and when athletes with tetraplegia/paresis were present in that sport, a further subdivision was made (Table 3). During a complete fitness assessment of Paralympic Athletes competing in Winter sports [4], representative of skill, power, intermittent endurance Athletes displayed mean power in the WAnT_10s, evaluated through the same electromagnetically braked ergometer (excalibur lode) and the same procedures, equalled to $3.6 \pm 1.13 \mathrm{~W} \mathrm{~kg}^{-1}$, $4.9 \pm 2.06,5.3 \pm 1.23,6.2 \pm 1.28$, respectively. Comparing these values with those of the present study, athletes competing in skill sports (sailing, table tennis, archery and shooting) had values $30 \%$ higher than those competing in curling. Athletes competing in intermittent sports (wheelchair fencing, basketball and tennis) had values $6 \%$ lower than Para Ice Hockey players. Endurance athletes (hand-bikers and swimmers) displayed values $12 \%$ lower than sitting Nordic skiers. Summer Paralympic Athletes competing in field events showed values 24\% lower than Alpine skiers. An arm cranking ergometer (angio lode) developed by the same brand of that of our study is routinely used to assess anaerobic fitness in wheelchair basketball players [37]. Comparing the WAnT_30s results of the latter Polish study with those of the present study, we found similar values in the mean power ( 4.25 vs. $4.49 \mathrm{~W} \mathrm{~kg}^{-1}$, respectively) while the peak values were much higher in the Polish wheelchair players $\left(7.95 \mathrm{~W} \mathrm{~kg}^{-1}\right)$ than in the Italian players $\left(6.28 \mathrm{~W} \mathrm{~kg}^{-1}\right)$. The excalibur lode ergometer has a fly wheel greater than that used in the arm cranking ergometer (angio lode) and used by the Polish scientists [37] because the torque values could significantly differ affecting the athletes' performance. Currently, to our best knowledge, there is no standardized method for determining the most appropriate torque value for the Wingate test protocol carried out on an electromagnetically braked ergometer in athletes with a locomotor impairment. We can suppose that little differences in the torque values and the different flywheels could affect the peak power in the WAnT. When comparing our data with the other figures present in the literature, it is important to take into consideration the possible differences between an electromagnetically braked ergometer and mechanically braked ergometers [56, 57]. Due to the discrepancies in absolute power values between differently braked ergometers [58], data from different devices (e.g., lode excalibur sport and monark cycle ergometer) cannot be used for interunit comparison. Anyway, it has been shown $[56,58,59]$ that the electromagnetically braked lode excalibur sport cycle ergometer provides reliable and highly correlated muscular 
power data in the WAnT compared with the Monark cycle ergometer.

The prediction results of the present study showed that the mean power assessed in the WAnT_10s and the functional class were able to predict about $90 \%$ of the total variance for the mean power assessed in the traditional WAnT_30s with a large effect size. Moreover, repeated cross-validation analysis, together with a Residual Diagnostic, highlighted the goodness-of-fit and the predictive power of the proposed model. Accordingly, the mean power assessed in the traditional WAnT_30s can be predicted accurately using data collected during the WAnT_10s in male Paralympic athletes. Beneke and colleagues in a traditional 30-s-long Wingate test carried out with the lower limbs on an electromagnetically braked ergometer [20] found that the total energy expenditure of the test was supplied for $19 \%$ by the aerobic metabolism and for 50 and $31 \%$ by the lactic and the alactic anaerobic metabolisms, respectively [20]. Lovell and collaborators [42] studied the different ATP contribution from the three energy metabolisms in an upper limb 30-s-long Wingate test carried out on a modified lode ergometer. They found that the percentages of energy contributions determined by the aerobic, anaerobic lactic, and anaerobic alactic energy systems were $11.4,60.3$ and $28.3 \%$, respectively. The authors [42] explain the slightly higher contribution from the lactic anaerobic metabolism considering how the upper body utilizes energy and responds to high-intensity exercise compared with the lower body. Bernardi and colleagues [5] in an all-out test carried out up to exhaustion (and lasting about $30 \mathrm{~s}$ ) in an arm cranking iso-power cadence independent ergometer (Ergometrics 800, Ergoline, Germany), test specifically designed for America's Cup Grinders, athletes with great muscle masses in the upper limbs, had found energy contributions from the 3 energy metabolism systems similar to the values found by Lovell and colleagues [42]. The total energy expended by the America's Cup Grinders was supplied for $15 \%$ by the aerobic metabolism and for $56 \%$ and $29 \%$ by the lactic and alactic anaerobic metabolisms, respectively [5]. Most muscle groups in the upper body contain a greater proportion of fast-twitch fibers [60] than those in the lower body, and because they extract less oxygen during exercise, they rely in a larger extent on anaerobic energy production during sprint exercise [61]. We can conclude that the prediction results of the present study can be justified by the great anaerobic contribution and the low aerobic contribution of both WAnT (10- and 30-s long). Furthermore, we can hypothesize a likely reduced contribution of the alactic anaerobic energy metabolism and relatively higher anaerobic glycolytic energy contribution in the WAnT_10s, typical of Athletes with impairment, who cannot rely on the lower part of the body. Gastin [17], reviewing the contributions in the ATP turnover of the energy systems in a 10-s-long all-out exercise carried out with the lower limbs, found that the alactic anaerobic metabolism accounted for about 50\% while the lactic anaerobic metabolism for about $45 \%$. These results show just a little difference, in terms of contribution of the lactic anaerobic metabolism, with respect to the three previously quoted studies (45\% in Gastin's Study [17] vs. about 55\%, as average of other 3 studies). The explanation of the similar contribution of anaerobic glycolytic metabolism between the all-out tests of different durations (10 vs. $30 \mathrm{~s}$ ) can be explained by the suppression of phosphofructokinase activity (key enzyme of the anaerobic glycolysis) determined by acidosis [16], which determines an ATP maximal turnover rate of this metabolism at around $13 \mathrm{~s}$ with a fast decrease thereafter. The suppression of the activity of this key enzyme suggests that an all-out test is not the most appropriate to evaluate the capacity of the lactic anaerobic metabolism [16]. Furthermore, the suppression of phosphofructokinase activity suggests the reason why the total energy provision is affected by the aerobic metabolism, explaining how an upper-body aerobic training, carried out at intensities close to the respiratory compensation point, in America's Cup grinders is able to determine significant improvements in the all-out test [62]. Considering that the anthropometric measures, which are proportional to the level of lesion/ impairment, are correlated with the alactic anaerobic capacity [42], taking into account the great contribution of the lactic anaerobic metabolism in an upper limb WAnT_10s, since the contribution of the alactic anaerobic metabolism is exhausted probably in the first $5 \mathrm{~s}$ for the small muscle mass available and the contribution of the aerobic metabolism is negligible, the predictability of the mean power assessed in the WAnT_30s from the values of mean power assessed in the WAnT_10s can be definitively explained.

The present study has some limitations that should be acknowledged. Our results have been limited to a small sample of male Paralympic athletes $(n=44)$ relatively to both the practiced sports and the ILG-A (the athletes with the highest level of impairment are 9). Further data are needed to confirm that a 10-s-long protocol is appropriate for assessing the anaerobic performance of those engaged in other levels of training and sports, in different age groups, and in particularly in female athletes. Furthermore, due to the heterogeneity of the considered population, future research in a larger sample size is needed to investigate different WAnT protocols according to the type and the severity of the physical impairment. However, it is important to underline that an inherent limitation when studying athletes with impairments is the enormous variability within the population itself resulting in an extremely heterogeneous sample. Furthermore, heterogeneous pools of high-level athletes with a physical impairment lead to large territorial spread for most studies, thus limiting the use of accurate measurement systems available by means of laboratory-based techniques. 
Accordingly, large samples of high-level athletes are difficult to recruit in this population.

In conclusion, the results presented herein offer important practical implications in Paralympic sports from the perspective of the anaerobic physical fitness evaluation. The WAnT_10s is accurate to assess peak power, is definitively appropriate to evaluate the alactic anaerobic metabolism and seems able to predict the mean power as traditionally evaluated through a WAnT_30s in male high-level athletes with various degrees of functional limitations allowing for a decrease in the mild to severe physical discomfort often associated with the longer traditional protocol. This may be especially important for those athletes with physical impairment involved in typical power sport disciplines, whose competitive efforts are based on the alactic and lactic anaerobic energy metabolism systems.

Acknowledgements The authors express their gratitude to the Paralympic athletes who participated in this study. This research is dedicated to Professor Marcello Faina who allowed this specific exercise testing during the "Health and fitness evaluations" carried out in Italy as pre-participation screening before the Paralympic Games.

Funding Open access funding provided by Università degli Studi di Verona within the CRUI-CARE Agreement.

\section{Compliance with ethical standards}

Conflict of interest The authors declare that they have no competing interests.

Ethical approval All procedures were in accordance with the ethical standards of the Institute of Sports Medicine and Science (ISMS) of the Italian National Olympic Committee - CONI (Rome, Italy), with the "Santa Lucia Foundation" Ethical Committee and with the 1964 Helsinki declaration and its later amendments or comparable ethical standards.

Informed consent Informed consent was obtained from all individual participants included in the study.

Open Access This article is licensed under a Creative Commons Attribution 4.0 International License, which permits use, sharing, adaptation, distribution and reproduction in any medium or format, as long as you give appropriate credit to the original author(s) and the source, provide a link to the Creative Commons licence, and indicate if changes were made. The images or other third party material in this article are included in the article's Creative Commons licence, unless indicated otherwise in a credit line to the material. If material is not included in the article's Creative Commons licence and your intended use is not permitted by statutory regulation or exceeds the permitted use, you will need to obtain permission directly from the copyright holder. To view a copy of this licence, visit http://creativecommons.org/licenses/by/4.0/.

\section{References}

1. Jee YS (2018) Participating in the Paralympic Games through exercise rehabilitation for individuals with disabilities. J Exerc Rehabil. 14(2):158-159. https://doi.org/10.12965/jer.1836188.094

2. Goosey-Tolfrey V (2010) Supporting the paralympic athlete: focus on wheeled sports. Disabil Rehabil 32(26):2237-2243. https://doi. org/10.3109/09638288.2010.491577

3. Bernardi M, Guerra E, Di Giacinto B, Di Cesare A, Castellano V, Bhambhani Y (2010) Field evaluation of paralympic athletes in selected sports: implications for training. Med Sci Sports Exerc 42(6):1200-1208. https://doi.org/10.1249/MSS.0b013e3181c67d8 2

4. Bernardi M, Carucci S, Faiola F, Egidi F, Marini C, Castellano V, Faina M (2012) Physical fitness evaluation of Paralympic winter sports sitting athletes. Clin J Sport Med 22(1):26-30. (Erratum in Clin J Sport Med 22(2):209)

5. Bernardi M, Quattrini FM, Rodio A, Fontana G, Madaffari A, Brugnoli M, Marchetti M (2007) Physiological characteristics of America's Cup sailors. J Sports Sci 25(10):1141-1152

6. Hutzler Y (1998) Anaerobic fitness testing of wheelchair users. Sports Med 25:101-113

7. Coutts KD (1992) Dynamics of wheelchair basketball. Med Sci Sports Exerc 24(2):231-234

8. Janssen TW, van Oers CA, van der Woude LH, Hollander AP (1994) Physical strain in daily life of wheelchair users with spinal cord injuries. Med Sci Sports Exerc 26(6):661-670. https://doi. org/10.1249/00005768-199406000-00002

9. Jacobs PL, Nash MS (2004) Exercise recommendations for individuals with spinal cord injury. Sports Med 34(11):727-751

10. Bernardi M, Janssen T, Bortolan L, Pellegrini B, Fischer G, Schena F (2013) Kinematics of cross-country sit skiing during a Paralympic race. J Electromyogr Kinesiol 23(1):94-101. https:// doi.org/10.1016/j.jelekin.2012.07.004

11. Fitts DL (1993) Cellular, molecular and metabolic basis of muscle fatigue. Am Physiol Soc 75:712

12. Bogdanis GC, Nevill ME, Lakomy HK, Boobis LH (1998) Power output and muscle metabolism during and following recovery from 10 and $20 \mathrm{~s}$ of maximal sprint exercise in humans. Acta Physiol Scand 163(3):261-272

13. Meyer R, Wieseman R (2006) The metabolic systems: control of ATP synthesis in skeletal muscle ACSM's advanced exercise physiology. Williams \& Wilkins, Lippincott, Philadelphia PA, p 370

14. Katch FI, Victor L (2010) Katch exercise physiology, 7th edn. Wolters Kluwer, USA

15. Greenhaff PL, Hultman E, Harris RC (1993) Carbohydrate metabolism. In: Poortmans JR (ed) Principles of exercise biochemistry. Karger, Basel, pp 89-136

16. Heck H, Schulz H, Bartmus U (2003) Diagnostics of anaerobic power and capacity. Eur J Sport Sci 3(3):1-23. https://doi. org/10.1080/17461390300073302

17. Gastin PB (2001) Energy system interaction and relative contribution during maximal exercise. Sports Med 31(10):725-741. https://doi.org/10.2165/00007256-200131100-00003

18. Parolin ML, Chesley A, Matsos MP, Sspriet LL, Jones NL, Heigenhause GJF (1999) Regulation of skeletal muscle glycogen phosphorylase and PDH during maximal intermittent exercise. Am J Physiol 277(5 Pt 1):E890-E900. https://doi.org/10.1152/ ajpendo.1999.277.5.E890

19. Green S, Dawson B (1993) Measurements of anaerobic capacities in humans. Definitions, limitations and unsolved problems. Sports Med 15(5):312-327. https://doi.org/10.2165/00007256199315050-00003 
20. Di Prampero PE, Feretti G (1999) The energetics of anaerobic muscle metabolism: a reappraisal of older and recent concepts. Respir Physiol 118(2-3):103-115. https://doi.org/10.1016/ S0034-5687(99)00083-3

21. Kavanagh MF, Jacobs I (1998) Breath-by-breath oxygen consumption during performance of the Wingate test. Can J Sport Sci 13:91-93

22. Beneke R, Pollmann C, Bleif I, Leithauser RM, Hutler M (2002) How anaerobic is the Wingate test for humans? Eur J Appl Physiol 87:388-392

23. Rodio A, Quattrini FM, Fattorini L, Egidi F, Faiola F, Pittiglio G (2008) Power output and metabolic response in multiple Wingate tests performed with arms [Potenza meccanica e risposta metabolica in Wingate-test multipli con le braccia]. Med Sport 61(1):21-28

24. Hutzler Y, Ochana S, Bolotin R, Kalina E (1998) Aerobic and anaerobic arm-cranking power outputs of males with lower limb impairments: relationship with sport participation intensity, age, impairment and functional classification. Spinal Cord 36:205-212

25. Jacobs PL, Mahoney ET, Johnson B (2003) Reliability of arm Wingate anaerobic testing in persons with complete paraplegia. Spinal Cord Med 26:141-144

26. Lees A, Arthur S (1988) An investigation into anaerobic performance of wheelchair athletes. Ergonomics 31(11):1529-1537

27. Coutts KD, Stogryn JL (1987) Aerobic and anaerobic power of Canadian wheelchair track athletes. Med Sci Sports Exerc 19(1):62-65

28. Hutzler Y (1993) Physical performance of elite wheelchair basketball players in armcranking ergometry and in selected wheeling tasks. Paraplegia 31:255-261

29. Jacobs PL, Johnson BM, Mahoney ET, Carter AB, Somarriba GA (2004) Effect of variable loading in the determination of upperlimb anaerobic power in persons with tetraplegia. J Rehabil Res Dev 41:9-14

30. Jacobs PL, Johnson B, Somarriba GA, Carter AB (2005) Reliability of upper extremity anaerobic power assessment in persons with tetraplegia. J Spinal Cord Med 28:109-113

31. Jacobs PL (2009) Effects of resistance and endurance training in persons with paraplegia. Med Sci Sports Exerc 41:992-997

32. Morgulec N, Kosmol A, Vanlandewijck Y, Hubner-Wozniak E (2005) Anaerobic performance of active and sedentary male individuals with quadriplegia. Adapt Phys Act Q 22:253-264

33. Nash MS, van de Ven I, van Elk N, Johnson BM (2007) Effects of circuit resistance training on fitness attributes and upper-extremity pain in middle-aged men with paraplegia. Arch Phys Med Rehabil 88:70-75

34. Porto YC, Almeida M, de Sá CK, Schwingel PA, Zoppi CC (2008) Anthropometric and physical characteristics of motor disabilited paralympic rowers. Res Sports Med 16(3):203-212. https://doi. org/10.1080/15438620802103437

35. Kressler J, Burns PA, Betancourt L, Nash MS (2014) Circuit training and protein supplementation in persons with chronic tetraplegia. Med Sci Sports Exerc 46:1277-1284

36. West CR, Campbell IG, Goosey-Tolfrey VL, Mason BS, Romer LM (2014) Effects of abdominal binding on field-based exercise responses in Paralympic athletes with cervical spinal cord injury. J Sci Med Sport 17(4):351-355. https://doi.org/10.1016/j.jsams .2013.06.001 (Epub 2013 Jul 21)

37. Runciman P, Derman W, Ferreira S, Albertus-Kajee Y, Tucker R (2015) A descriptive comparison of sprint cycling performance and neuromuscular characteristics in able-bodied athletes and paralympic athletes with cerebral palsy. Am J Phys Med Rehabil 94(1):28-37. https://doi.org/10.1097/PHM.0000000000000136

38. Basar S, Ergun N, Yigiter Bayramlar K (2013) A comparative study of muscle strength and anaerobic power of the young
National and National Junior Wheelchair Basketball players. Turkish J Phys Med Rehabil 59:325-329

39. Marszałek J, Kosmol A, Morgulec-Adamowicz N, Mróz A, Gryko K, Klavina A, Skucas K, Navia JA, Molik B (2019) Laboratory and non-laboratory assessment of anaerobic performance of Elite male wheelchair basketball athletes. Front Psychol 10:514. https ://doi.org/10.3389/fpsyg.2019.00514

40. Goosey-Tolfrey V, Castle P, Webborn N, Abel T (2006) Aerobic capacity and peak power output of elite quadriplegic games players. Br J Sports Med 40:684-687

41. Maud PJ, Shultz BB (1989) Norms for the Wingate anaerobic test with comparison to another similar test. Res Q Exerc Sport 60(2):144-151

42. Medbø JI (1985) Tabata I (1993) Anaerobic energy release in working muscle during $30 \mathrm{~s}$ to 3 min of exhausting bicycling. J Appl Physiol 75(4):1654-1660

43. Jacobs I, Bar-Or O, Karlsson J, Dotan R, Tesch P, Kaiser P, Inbar $\mathrm{O}$ (1982) Changes in muscle metabolites in females with 30-s exhaustive exercise. Med Sci Sports Exerc 14:457-460

44. Lovell D, Kerr A, Wiegand A, Solomon C, Harvey L, McLellan C (2013) The contribution of energy systems during the upper body Wingate anaerobic test. Appl Physiol Nutr Metabol 38(2):216219. https://doi.org/10.1139/apnm-2012-0101

45. Smith JC, Hill DW (1991) Contribution of energy systems during a Wingate power test. Br J Sports Med 25(4):196-199

46. International Paralympic Committee. Explanatory guide to Paralympic classification. Paralympic summer sports. Sep 2015. https ://www.paralympic.org/sites/default/files/document/1509151708 06821_2015_09_15\%2BExplanatory\%2Bguide\%2BClassificatio n_summer\%2BFINAL\%2B_5.pdf.

47. Pelliccia A, Quattrini FM, Squeo MR, Caselli S, Culasso F, Link MS, Spataro A, Bernardi M (2016) Cardiovascular diseases in Paralympic athletes. Br J Sports Med 50(17):1075-1080. https:// doi.org/10.1136/bjsports-2015-095867

48. Bernardi M, Guerra E, Rodio A, Dante D, Castellano V, Peluso I, Schena F, Bhambhani Y (2020) Assessment of exercise stroke volume and its prediction from oxygen pulse in paralympic athletes with locomotor impairments: cardiac long-term adaptations are possible. Front Physiol 10:1451. https://doi.org/10.3389/fphys .2019.01451

49. Egidi F, Faiola F, Guerra E, Marini C, Sardella F, Bernardi M (2009) Sport for disabled individuals: from rehabilitation to Paralympic games. Med Sport 62(4):597-601

50. Rosenberger WF, Lachin JM (2016) Randomization in clinical trials theory and practice, 2nd edn. Wiley, Hoboken

51. Bernardi M, Schena F (2011) Preparation for the Paralympic winter games: cold, altitude. In: Vanlandewijck YC, Thompson WR (eds) Handbook of sports medicine and science-the Paralympic athlete, vol 13. Wiley-Blackwell, Chichester, pp 231-248

52. Cohen J (1988) Statistical power analysis for the behavioral sciences, 2nd edn. Lawrence Erl baum Associates, Hillsdale (NJ)

53. Faul F, Erdfelder E, Buchner A, Lang AG (2009) Statistical power analyses using $\mathrm{G}^{*}$ Power 3.1: tests for correlation and regression analyses. Behav Res Methods 41(4):1149-1160. https://doi. org/10.3758/BRM.41.4.1149PMID19897823

54. Price M (2010) Energy expenditure and metabolism during exercise in persons with a spinal cord injury. Sports Med 40:681-696. https://doi.org/10.2165/11531960-000000000-00000

55. Baumgart JK, Brurok B, Sandbakk Ø (2018) Peak oxygen uptake in Paralympic sitting sports: a systematic literature review, metaand pooled-data analysis. PLoS ONE 13:e0192903. https://doi. org/10.1371/journal.pone.0192903

56. Serresse O, Lortie G, Bouchard C, Boulay MR (1988) Estimation of the contribution of the various energy systems during maximal work of short duration. Int J Sports Med 9:456-460 
57. Laurent CM Jr, Meyers MC, Robinson CA, Green JM (2007) Cross-validation of the 20- versus 30-s Wingate anaerobic test. Eur J Appl Physiol 100(6):645-651

58. Micklewright D, Alkhatib A, Beneke R (2006) Mechanically versus electromagnetically braked cycle ergometer: performance and energy cost of the Wingate anaerobic test. Eur J Appl Physiol 96(6):748-751. https://doi.org/10.1007/s00421-006-0145-5

59. Dotan R (2006) The Wingate anaerobic test's past and future and the compatibility of mechanically versus electro-magnetically braked cycle-ergometers. Eur J Appl Physiol 98(1):113-116. https ://doi.org/10.1007/s00421-006-0251-4

60. Lunn WR, Axtell RS (2019) Validity and reliability of the lode excalibur sport cycle ergometer for the Wingate anaerobic test. J Strength Cond Res. https://doi.org/10.1519/JSC.0000000000 003211.10.1519

61. Earnest CP, Wharton RP, Church TS, Lucia A (2005) Reliability of the lode excalibur sport ergometer and applicability to computrainer electromagnetically braked cycling training device. Med Sci Sports Exerc 19(2):44-348. https://doi.org/10.1519/R-15714 .1

62. Sanchís-Moysi J, Idoate F, Olmedillas H, Guadalupe-Grau A, Alayon S, Carreras A et al (2010) The upper extremity of the professional tennis player: muscle volumes, fiber-type distribution and muscle strength. Scand J Med Sci Sports 20:524-534. https:// doi.org/10.1111/j.1600-0838.2009.00969.x

63. Zinner C, Morales-Alamo D, Ørtenblad N, Larsen FJ, Schiffer TA, Willis SJ, Gelabert-Rebato M, Perez-Valera M, Boushel R, Calbet JAL, Holmberg HC (2016) The physiological mechanisms of performance enhancement with sprint interval training differ between the upper and lower extremities in humans. Front Physiol 7:426. https://doi.org/10.3389/fphys.2016.00426

64. Adami PE, Delussu AS, Squeo MR, Corsi L, Rodio A, Fattorini L, Quattrini FM, Bernardi M (2015) Upper limb aerobic training improves aerobic fitness and all-out performance of America's Cup grinders. Eur J Sports Sci 15(3):235-241. https://doi. org/10.1080/17461391.2014.971878

Publisher's Note Springer Nature remains neutral with regard to jurisdictional claims in published maps and institutional affiliations. 